\title{
Oscillatory Behavior of Second Order Nonlinear Differential Equations with a Sublinear Neutral Term
}

Said R. Grace ${ }^{a}$ and John R. Graef ${ }^{b}$

${ }^{a}$ Cairo University, Department of Engineering Mathematics, Faculty of Engineering

Orman, 12221 Giza, Egypt

${ }^{b}$ Department of Mathematics, University of Tennessee at Chattanooga

Chattanooga, $37403 \mathrm{TN}$, USA

E-mail(corresp.): John-Graef@utc.edu

E-mail: saidgrace@yahoo.com

Received August 1, 2017; revised January 12, 2018; accepted January 26, 2018

Abstract. The authors establish some new criteria for the oscillation of solutions of second order nonlinear differential equations with a sublinear neutral term by reducing the equation to a linear one. Their results are illustrated with an example.

Keywords: oscillation, second order, neutral differential equations, sublinear neutral term.

AMS Subject Classification: 34K11; 34C10; 34C15.

\section{Introduction}

This paper deals with the oscillatory behavior of all solutions of the nonlinear second order differential equation with a sublinear neutral term

$$
\left(a(t)\left(x(t)+p(t) x^{\alpha}(\sigma(t))\right)^{\prime}\right)^{\prime}+q(t) x^{\beta}(\tau(t))=0,
$$

where we assume that

(i) $\alpha$ and $\beta$ are the ratios of positive odd integers with $0<\alpha<1$;

(ii) $a, p, q:\left[t_{0}, \infty\right) \rightarrow \mathbb{R}^{+}$are continuous functions with

$$
\int_{t_{0}}^{\infty} 1 / a(s) d s<\infty ;
$$

Copyright (c) 2018 The Author(s). Published by VGTU Press

This is an Open Access article distributed under the terms of the Creative Commons Attribution License (http://creativecommons.org/licenses/by/4.0/), which permits unrestricted use, distribution, and reproduction in any medium, provided the original author and source are credited. 
(iii) $\tau, \sigma:\left[t_{0}, \infty\right) \rightarrow \mathbb{R}$ are continuous functions with $\tau(t), \sigma(t) \leq t, \tau^{\prime}(t)>0$, and $\tau(t), \sigma(t) \rightarrow \infty$ as $t \rightarrow \infty$.

In recent years, there has been much research activity concerning the oscillation and nonoscillation of solutions of various differential equations, and we refer the reader to $[2,3,4,5,6,7,8,10]$ for recent references. However, there are few results dealing with the oscillation of second order differential equations with a sublinear neutral term; see [1] as one example.

In establishing some new criteria for the oscillation of solutions of such equations, our approach is, in some sense, to reduce the equation to a linear one. We let

$$
A(t)=\int_{t}^{\infty} 1 / a(s) d s
$$

For convenience we set $y(t)=x(t)+p(t) x^{\alpha}(\sigma(t))$. By a solution of equation (1.1) we mean a function $x(t)$ where both the quasi-derivatives $a(t) y^{\prime}(t)$ and $\left(a(t) y^{\prime}(t)\right)^{\prime}$ are continuous on $\left[T_{x}, \infty\right), T_{x} \geq t_{0}$, and which satisfies Equation (1.1) on $\left[T_{x}, \infty\right)$. We consider only those solutions $x(t)$ of (1.1) that are continuable, that is, they satisfy $\sup \{|x(t)|: t \geq T\}>0$ for all $T \geq T_{x}$. A solution of (1.1) is said to be oscillatory if it has arbitrarily large zeros, and nonoscillatory otherwise. Equation (1.1) will be said to be oscillatory if all its solutions are oscillatory.

\section{Main results}

We will need the following lemma in the proofs of our results.

Lemma 1. ( [9]) If $X$ and $Y$ are nonnegative, then

$$
\begin{aligned}
& X^{\lambda}+(\lambda-1) Y^{\lambda}-\lambda X Y^{\lambda-1} \geq 0 \quad \text { if } \lambda>1, \\
& X^{\lambda}-(1-\lambda) Y^{\lambda}-\lambda X Y^{\lambda-1} \leq 0 \quad \text { if } \lambda<1,
\end{aligned}
$$

where equality holds if and only if $X=Y$.

In an effort to somewhat simplify our notation, for any positive continuous functions $p_{1}, p_{2}:\left[t_{0}, \infty\right) \rightarrow \mathbb{R}^{+}$, we set:

$$
\begin{aligned}
& g_{1}(t)=(1-\alpha) \alpha^{\frac{\alpha}{1-\alpha}} p_{1}^{\frac{\alpha}{\alpha-1}}(t) p^{\frac{1}{1-\alpha}}(t), \quad P(t)=\left(1-p_{1}(t)-g_{1}(t) / A(t)\right), \\
& g_{2}(t)=(\beta-1) \beta^{\frac{\beta}{1-\beta}}\left[q(t) P^{\beta}(\tau(t))\right]^{\frac{1}{1-\beta}} p_{2}^{\frac{\beta}{\beta-1}}(t), \\
& P^{*}(t)=1-\left(p_{1}(t)+\frac{g_{1}(t)}{A^{2}(\sigma(t))}\right)\left(\frac{A(\sigma(t))}{A(t)}\right), \\
& g_{2}^{*}(t)=(\beta-1) \beta^{\frac{\beta}{1-\beta}}\left[q(t)\left(P^{*}(t)\right)^{\beta}\right]^{\frac{1}{1-\beta}} p_{2}^{\frac{\beta}{\beta-1}}(t), \\
& Q^{*}(t)=\left(p_{2}(t)-g_{2}(t) / A(\tau(t))\right), \quad Q_{1}(t)=p_{2}(t)-g_{2}^{*}(t) / A^{2}(t) .
\end{aligned}
$$

We now present our first oscillation result, it is for the case where $\beta>1$. 
Theorem 1. Let $\beta>1$ and conditions (i)-(iii) and (1.2) hold. Assume that there are positive continuous functions $p_{1}, p_{2}:\left[t_{0}, \infty\right) \rightarrow \mathbb{R}^{+}$such that $P(t)$, $Q^{*}(t)$, and $Q_{1}(t)$ are positive for $t \geq t_{0}$. If there exists a positive function $\rho \in C^{1}\left(\left[t_{0}, \infty\right), \mathbb{R}\right)$ such that

$$
\limsup _{t \rightarrow \infty} \int_{t_{0}}^{t}\left[\rho(s) Q^{*}(s)-\frac{a(\tau(s))\left(\rho^{\prime}(s)\right)^{2}}{4 \rho(s) \tau^{\prime}(s)}\right] d s=\infty
$$

and

$$
\limsup _{t \rightarrow \infty} \int_{t_{0}}^{t}\left[A(s) Q_{1}(s)-\frac{1}{4 a(s) A(s)}\right] d s=\infty,
$$

then equation (1.1) is oscillatory.

Proof. Let $x(t)$ be a nonoscillatory solution of equation (1.1), say $x(t)>0$, $x(\tau(t))>0$, and $x(\sigma(t))>0$ for $t \geq t_{1}$ for some $t_{1} \geq t_{0}$. It is easy to see that $y(t)>0$ for $t \geq t_{1}$ and that equation (1.1) can be written as

$$
\left(a(t) y^{\prime}(t)\right)^{\prime}+q(t) x^{\beta}(\tau(t))=0 .
$$

From this we see that $a(t) y^{\prime}(t)$ is decreasing and so either (I) $y^{\prime}(t)>0$ or (II) $y^{\prime}(t)<0$ for $t \geq t_{2}$ for some $t_{2} \geq t_{1}$.

First we consider Case (I). From the definition of $y(t)$ we can write

$$
x(t)=y(t)-\left[p(t) x^{\alpha}(\sigma(t))-p_{1}(t) x(\sigma(t))\right]-p_{1}(t) x(\sigma(t)) .
$$

Now applying (2.2) with

$$
\lambda=\alpha, \quad X=p^{1 / \alpha} x \quad \text { and } \quad Y=\left(p_{1} p^{\frac{-1}{\alpha}} / \alpha\right)^{\frac{1}{\alpha-1}}
$$

we obtain

$$
p(t) x^{\alpha}(t)-p_{1}(t) x(t) \leq(1-\alpha) \alpha^{\frac{\alpha}{1-\alpha}} p_{1}^{\frac{\alpha}{\alpha-1}}(t) p^{\frac{1}{1-\alpha}}(t):=g_{1}(t) \text { for } t \geq t_{2} .
$$

Since $x(t) \leq y(t)$, we have

$$
\begin{gathered}
x(t) \geq y(t)-p_{1}(t) x(\sigma(t))-g_{1}(t) \geq y(t)-p_{1}(t) y(\sigma(t))-g_{1}(t) \\
\geq y(t)-p_{1}(t) y(t)-g_{1}(t) .
\end{gathered}
$$

Since $y(t)$ is positive and increasing and $A(t)$ is positive and decreasing to zero, there exists $t_{3} \geq t_{2}$ such that

$$
y(t) \geq A(t) \text { for } t \geq t_{3}
$$

Using (2.7) in (2.6), we have

$$
x(t) \geq\left(1-p_{1}(t)-\frac{1}{A(t)} g_{1}(t)\right) y(t):=P(t) y(t),
$$

and substituting this into equation (1.1) gives

$$
\left(a(t) y^{\prime}(t)\right)^{\prime}+q(t) P^{\beta}(\tau(t)) y^{\beta}(\tau(t)) \leq 0 .
$$


Hence,

$$
\begin{aligned}
\left(a(t) y^{\prime}(t)\right)^{\prime} & \leq-q(t) P^{\beta}(\tau(t)) y^{\beta}(\tau(t))=-q(t) P^{\beta}(\tau(t)) y^{\beta}(\tau(t)) \\
& =\left[p_{2}(t) y(\tau(t))-q(t) P^{\beta}(\tau(t)) y^{\beta}(\tau(t))\right]-p_{2}(t) y(\tau(t)) .
\end{aligned}
$$

If we now apply (2.1) with

$$
\begin{aligned}
& \lambda=\beta, \quad X=\left[q(t) P^{\beta}(\tau(t))\right]^{1 / \beta} y(\tau(t)), \\
& Y=\left(\frac{1}{\beta} p_{2}(t)\left[q(t) P^{\beta}(\tau(t))\right]^{-1 / \beta}\right)^{1 /(\beta-1)},
\end{aligned}
$$

we have

$$
\begin{aligned}
p_{2}(t) y(\tau(t))-q(t) P^{\beta} & (\tau(t)) y^{\beta}(\tau(t)) \\
& \leq(\beta-1) \beta^{\frac{\beta}{1-\beta}}\left[q(t) P^{\beta}(\tau(t))\right]^{\frac{1}{1-\beta}} p_{2}^{\frac{\beta}{\beta-1}}(t)=g_{2}(t)
\end{aligned}
$$

for $t \geq t_{3}$. Using (2.10) in (2.9), we obtain

$$
\begin{aligned}
\left(a(t) y^{\prime}(t)\right)^{\prime} & \leq-q(t) P^{\beta}(\tau(t)) y^{\beta}(\tau(t)) \\
& =g_{2}(t)-p_{2}(t) y(\tau(t))=\left(\frac{g_{2}(t)}{y(\tau(t))}-p_{2}(t)\right) y(\tau(t)),
\end{aligned}
$$

so from (2.7),

$$
\left(a(t) y^{\prime}(t)\right)^{\prime} \leq-\left(p_{2}(t)-\frac{g_{2}(t)}{y(\tau(t))}\right) y(\tau(t))=-Q^{*}(t) y(\tau(t)) .
$$

Define the function

$$
w(t)=\rho(t) \frac{a(t) y^{\prime}(t)}{y(\tau(t))}, \quad \text { for } t \geq t_{3} .
$$

Then $w(t)>0$ for $t \geq t_{3}$ and

$$
w^{\prime}(t)=\rho^{\prime}(t) \frac{a(t) y^{\prime}(t)}{y(\tau(t))}+\rho(t) \frac{\left(a(t) y^{\prime}(t)\right)^{\prime}}{y(\tau(t))}-\rho(t) \frac{a(t) y^{\prime}(t) y^{\prime}(\tau(t)) \tau^{\prime}(t)}{y^{2}(\tau(t))} .
$$

Since $a(t) y^{\prime}(t)$ is positive and nonincreasing,

$$
a(t) y^{\prime}(t) \leq a(\tau(t)) y^{\prime}(\tau(t)) .
$$

Using this inequality, (2.11) and completing the square on the first and third terms, we see that

$$
w^{\prime}(t) \leq-\rho(t) Q^{*}(t)+\frac{a(\tau(t))\left(\rho^{\prime}(t)\right)^{2}}{4 \rho(t) \tau^{\prime}(t)} .
$$

Integrating the last inequality from $t_{3}$ to $t$ gives

$$
\int_{t_{3}}^{t}\left[\rho(s) Q^{*}(s)-\frac{a(\tau(s))\left(\rho^{\prime}(s)\right)^{2}}{4 \rho(s) \tau^{\prime}(s)}\right] d s \leq w\left(t_{2}\right),
$$


which contradicts condition (2.3).

Next, we consider Case (II), so suppose $y^{\prime}(t)<0$ for $t \geq t_{1}$. Define the function $v(t)$ by

$$
v(t)=\frac{a(t) y^{\prime}(t)}{y(t)} \quad \text { for } t \geq t_{1} ;
$$

then $v(t)<0$ for $t \geq t_{1}$. It is easy to see that

$$
y^{\prime}(s) \leq \frac{a(t)}{a(s)} y^{\prime}(t) \text { for } s \geq t,
$$

and an integration yields

$$
y(u)-y(t) \leq a(t) y^{\prime}(t)\left(\int_{t}^{u} \frac{d s}{a(s)}\right) .
$$

Taking the limit as $u \rightarrow \infty$, we obtain

$$
\frac{a(t) y^{\prime}(t)}{y(t)} A(t) \geq-1
$$

that is,

$$
v(t) A(t) \geq-1
$$

On the other hand, from (2.13),

$$
\left(\frac{y(t)}{A(t)}\right)^{\prime} \geq 0 \text { for } t \geq t_{1}
$$

Since $y(t) / A(t)$ is positive and increasing and $A(t)$ is positive and decreasing to zero, there exists $t_{2} \geq t_{1}$ such that

$$
y(t) \geq A^{2}(t) \quad \text { for } t \geq t_{2} .
$$

As in Case I above, (2.5) holds, and using (2.16), we see that

$$
\begin{aligned}
x(t) & \geq y(t)-p_{1}(t) y(\sigma(t))-g_{1}(t) \\
& \geq y(t)-\left[p_{1}(t)+g_{1}(t) / A^{2}(\sigma(t))\right] y(\sigma(t)) .
\end{aligned}
$$

Using (2.15) in (2.17), we obtain

$$
x(t) \geq\left[1-\left(p_{1}(t)+\frac{g_{1}(t)}{A^{2}(\sigma(t))}\right)\left(\frac{A(\sigma(t))}{A(t)}\right)\right] y(t)=P^{*}(t) y(t),
$$

and using (2.18) in (1.1), we have

$$
\begin{aligned}
\left(a(t) y^{\prime}(t)\right)^{\prime} & =-q(t) x^{\beta}\left(\tau(t) \leq-q(t)\left(P^{*}(t)\right)^{\beta} y^{\beta}(t)=-q(t)\left(P^{*}(t)\right)^{\beta} y^{\beta}(t)\right. \\
& =\left(p_{2}(t) y(t)-q(t)\left(P^{*}(t)\right)^{\beta} y^{\beta}(t)\right)-p_{2}(t) y(t) .
\end{aligned}
$$

Next, applying $(2.1)$ with $\lambda=\beta, X=\left(q(t)\left(P^{*}(t)\right)^{\beta}\right)^{1 / \beta} y(t)$ and

$$
Y=\left(\frac{1}{\beta} p_{2}(t)\left(q(t)\left(P^{*}(t)\right)^{\beta}\right)^{-1 / \beta}\right)^{1 /(\beta-1)},
$$


then we have

$$
\begin{aligned}
& p_{2}(t) y(t)-q(t)\left(P^{*}(t)\right)^{\beta} y^{\beta}(t) \\
& \quad \leq(\beta-1) \beta^{\frac{\beta}{1-\beta}}\left(q(t)\left(P^{*}(t)\right)^{\beta}\right)^{\frac{1}{1-\beta}} p_{2}^{\frac{\beta}{\beta-1}}(t)=g_{2}^{*}(t) \text { for } t \geq t_{2} .
\end{aligned}
$$

Combining (2.16), (2.20) and (2.19) gives

$$
\begin{aligned}
\left(a(t) y^{\prime}(t)\right)^{\prime} & \leq q(t)\left(P^{*}(t)\right)^{\beta} y^{\beta}(t) \\
& =g_{2}^{*}(t)-p_{2}(t) y(t)=-\left(p_{2}(t)-\frac{g_{2}^{*}(t)}{y(t)}\right) y(t) \\
& \leq-\left(p_{2}(t)-\frac{g_{2}^{*}(t)}{A^{2}(t)}\right) y(t)=-Q_{1}(t) y(t) .
\end{aligned}
$$

Differentiating (2.12), we obtain

$$
v^{\prime}(t)=\frac{\left(a(t) y^{\prime}(t)\right)^{\prime}}{y(t)}-\frac{v^{2}(t)}{a(t)}
$$

so from (2.21) and (2.22), we have

$$
v^{\prime}(t) \leq-Q_{1}(t)-\frac{v^{2}(t)}{a(t)}
$$

If we then multiply both sides of $(2.23)$ by $A(t)$ and integrate the resulting inequality from $t_{2}$ to $t$, we see that

$$
A(t) v(t)-A\left(t_{2}\right) v\left(t_{2}\right)+\int_{t_{2}}^{t} Q_{1}(s) A(s) d s+\int_{t_{2}}^{t} \frac{v(s)}{a(s)} d s+\int_{t_{2}}^{t} A(s) \frac{v^{2}(s)}{a(s)} d s \leq 0 .
$$

Completing the square on the last two terms on the right hand side and using (2.14) yields

$$
\int_{t_{2}}^{t}\left[A(s) Q_{1}(s)-\frac{1}{4 a(s) A(s)}\right] d s \leq 1+A\left(t_{2}\right) v\left(t_{2}\right)<\infty,
$$

which contradicts (2.4). This completes the proof of the theorem.

Next, we establish another new oscillation result for equation (1.1) with $\beta>1$.

Theorem 2. Let $\beta>1$ and conditions (i)-(iii) and (1.2) hold. Assume that there is a positive continuous function $p_{1}:\left[t_{0}, \infty\right) \rightarrow \mathbb{R}^{+}$such that $P(t)$ and $P^{*}(t)$ are positive for $t \geq t_{0}$. If there exists a positive function $\rho \in$ $C^{1}\left(\left[t_{0}, \infty\right), \mathbb{R}\right)$ such that

$$
\begin{aligned}
& \limsup _{t \rightarrow \infty} \int_{t_{0}}^{t}\left[\rho(s) q(s) A^{\beta-1}(\tau(s)) P^{\beta}(\tau(s))-\frac{a(\tau(s))\left(\rho^{\prime}(s)\right)^{2}}{4 \rho(s) \tau^{\prime}(s)}\right] d s=\infty, \\
& \limsup _{t \rightarrow \infty} \int_{t_{0}}^{t}\left[q(s) A^{2 \beta-1}(s)\left(P^{*}(s)\right)^{\beta}-\frac{1}{4 a(s) A(s)}\right] d s=\infty,
\end{aligned}
$$

then equation (1.1) is oscillatory. 
Proof. Let $x(t)$ be a nonoscillatory solution of (1.1), say $x(t)>0, x(\tau(t))>0$, and $x(\sigma(t))>0$ for $t \geq t_{1}$ for some $t_{1} \geq t_{0}$. Proceeding as in the proof of Theorem 1, we see that either Case (I) or Case (II) holds for $t \geq t_{2}$ for some $t_{2} \geq t_{1}$. As in the proof of Case (I) in Theorem 1, we again arrive at (2.8). Now, using (2.7) in (2.8), we have

$$
\left(a(t) y^{\prime}(t)\right)^{\prime}+q(t) A^{\beta-1}(\tau(t)) P^{\beta}(\tau(t)) y(\tau(t) \leq 0 .
$$

The rest of the proof in this case is similar to that of Case (I) in Theorem 1 and hence is omitted.

Next, we proceed as in the proof of Case (II) in Theorem 1 to obtain (2.19). Using (2.16) in (2.19) we have

$$
\left.\left(a(t) y^{\prime}(t)\right)^{\prime}=-q(t)\right) x^{\beta}(\tau(t)) \leq-q(t) A^{2(\beta-1)}(t)\left(P^{*}(t)\right)^{\beta} y(t) .
$$

The remainder of the proof is similar to that of Case (II) in Theorem 1 and we omit the details.

If $\beta=1$, we immediately have the following oscillation result for equation (1.1).

Theorem 3. Let $\beta=1$ and conditions (i)-(iii) and (1.2) hold. Assume that there is a positive continuous function $p_{1}:\left[t_{0}, \infty\right) \rightarrow \mathbb{R}^{+}$such that $P(t)>0$ and $P^{*}(t)>0$ for $t \geq t_{0}$. If there exists a positive function $\rho(t) \in C^{1}\left(\left[t_{0}, \infty\right), \mathbb{R}\right)$ such that

$$
\begin{aligned}
& \limsup _{t \rightarrow \infty} \int_{t_{0}}^{t}\left[\rho(s) q(s) P(\tau(s))-\frac{a(\tau(s))\left(\rho^{\prime}(s)\right)^{2}}{4 \rho(s) \tau^{\prime}(s)}\right] d s=\infty, \\
& \limsup _{t \rightarrow \infty} \int_{t_{0}}^{t}\left[A(s) q(s) P^{*}(s)-\frac{1}{4 a(s) A(s)}\right] d s=\infty,
\end{aligned}
$$

then equation (1.1) is oscillatory.

Next, we establish an oscillation result in case $0<\beta<1$.

Theorem 4. Let $0<\beta<1$ and conditions (i)-(iii) and (1.2) hold. Assume that there is a positive continuous function $p_{1}:\left[t_{0}, \infty\right) \rightarrow \mathbb{R}^{+}$such that $P(t)$ and $P^{*}(t)$ are positive for $t \geq t_{0}$. If there exists a positive function $\rho \in C^{1}\left(\left[t_{0}, \infty\right), \mathbb{R}\right)$ such that

$$
\begin{aligned}
& \limsup _{t \rightarrow \infty} \int_{t_{0}}^{t}\left[\rho(s) \frac{q(s) P^{\beta}(\tau(s))}{K^{1-\beta}}-\frac{a(\tau(s))\left(\rho^{\prime}(s)\right)^{2}}{4 \rho(s) \tau^{\prime}(s)}\right] d s=\infty, \\
& \limsup _{t \rightarrow \infty} \int_{t_{0}}^{t}\left[A(s) \frac{q(s)\left(P^{*}(s)\right)^{\beta}}{K^{1-\beta}}-\frac{1}{4 a(s) A(s)}\right] d s=\infty
\end{aligned}
$$

for every constant $K>0$, then equation (1.1) is oscillatory.

Proof. Let $x(t)$ be a nonoscillatory solution of (1.1), say $x(t)>0, x(\tau(t))>0$, $x(\sigma(t))>0$ and $y(t)>0$ for $t \geq t_{1}$ for some $t_{1} \geq t_{0}$. Proceeding as in the proof of Theorem 1, we again arrive at the two cases: 
for some $t_{2} \geq t_{1}$.

$$
\text { (I) } y^{\prime}(t)>0 \text { or (II) } y^{\prime}(t)<0 \text { for } t \geq t_{2}
$$

In considering Case (I) we obtain (2.8) as before. Since $a(t) y^{\prime}(t)$ is nonincreasing on $\left[t_{2}, \infty\right)$, there exists a constant $C>0$ such that

$$
a(t) y^{\prime}(t)<C \quad \text { for } t \geq t_{2} \text {. }
$$

Integrating from $t_{2}$ to $t$, in view of (1.2), we have

$$
y(t) \leq C \int_{t_{2}}^{t} \frac{1}{a(s)} d s+y\left(t_{2}\right) \leq K \quad \text { for } t \geq t_{2}
$$

for some constant $K>0$. Using (2.30) in (2.8) we obtain

$$
\left(a(t) y^{\prime}(t)\right)^{\prime}+\frac{q(t) P^{\beta}(\tau(t))}{K^{1-\beta}} y(\tau(t)) \leq\left(a(t) y^{\prime}(t)\right)^{\prime}+q(t) P^{\beta}(\tau(t)) \frac{y(\tau(t))}{y^{1-\beta}(\tau(t))} \leq 0 .
$$

The remainder of the proof in this case is similar to that of Theorem 1 and hence is omitted.

To prove the theorem if Case (II) holds, we proceed as in the proof of Case (II) in Theorem 1, and again obtain (2.19). Using (2.30) in (2.19) gives

$$
\left(a(t) y^{\prime}(t)\right)^{\prime} \leq-\frac{q(t)\left(P^{*}(t)\right)^{\beta}}{K^{1-\beta}} y(t) .
$$

The remainder of the proof is similar to that of the corresponding part of the proof of Theorem 1 and hence is omitted.

To illustrate our results we have the following example.

Example 1. Consider the differential equation with a sublinear neutral term

$$
\left(t^{2}\left(x(t)+\frac{1}{t^{2}} x^{1 / 3}(t / 2)\right)^{\prime}\right)^{\prime}+t^{\gamma} x^{\beta}(t / 2)=0, \quad t \geq 4
$$

Here, $\alpha=1 / 3, \beta$ is the ratio of positive odd integers, $a(t)=t^{2}, \tau(t)=\sigma(t)=$ $t / 2, p(t)=1 / t^{2}$ and $q(t)=t^{\gamma}, \gamma \in \mathbb{R}$.

It is easy to see that (1.2) holds and $A(t)=1 / t$. Letting $p_{1}(t)=p(t)$, we see that $g_{1}(t)=2 /\left(3 \sqrt{3} t^{2}\right)$, and so

$$
\frac{1}{2}<P(t)=\left(1-\frac{1}{t^{2}}-\frac{2}{3 \sqrt{3} t}\right)<1 \text { and } \frac{1}{2} \leq P^{*}(t)=\left(\frac{3 \sqrt{3}-1}{3 \sqrt{3}}-\frac{2}{t^{2}}\right)<1 .
$$

Letting $\beta=1 / 3, \gamma>0$ and $\rho(t)=t$, we see that

$$
\begin{aligned}
\int_{t_{0}}^{t}\left[K^{\beta-1} \rho(s) q(s) P^{\beta}(\tau(s))-\frac{a(\tau(s))\left(\rho^{\prime}(s)\right)^{2}}{4 \rho(s) \tau^{\prime}(s)}\right] d s & \\
& =\int_{4}^{t}\left[K^{-\frac{2}{3}} s^{\gamma+1}\left(1-\frac{4}{s^{2}}-\frac{4}{3 \sqrt{3} s}\right)^{1 / 3}-\frac{s}{8}\right] d s \rightarrow \infty \text { as } t \rightarrow \infty
\end{aligned}
$$


and

$$
\begin{aligned}
& \int_{t_{0}}^{t}\left[K^{\beta-1} A(s) q(s)\left(P^{*}(s)\right)^{\beta}-\frac{1}{4 a(s) A(s)}\right] d s \\
&=\int_{4}^{t}\left[K^{-\frac{2}{3}} s^{\gamma-1}\left(\frac{3 \sqrt{3}-1}{3 \sqrt{3}}-\frac{2}{s^{2}}\right)^{1 / 3}-\frac{1}{4 s}\right] d s \rightarrow \infty \text { as } t \rightarrow \infty
\end{aligned}
$$

for every $K>0$, i.e., conditions (2.28) and (2.29) of Theorem 4 are satisfied. Hence equation (2.31) is oscillatory. Now, we let $\beta=1, \gamma>0$ and $\rho(t)=t$. Then conditions (2.26) and (2.27) become

$$
\begin{aligned}
& \int_{4}^{t}\left[s^{\gamma+1}\left(1-\frac{4}{s^{2}}-\frac{4}{3 \sqrt{3} s}\right)-\frac{s}{8}\right] d s \rightarrow \infty \text { as } t \rightarrow \infty, \\
& \int_{4}^{t}\left[s^{\gamma-1}\left(\frac{3 \sqrt{3}-1}{3 \sqrt{3}}-\frac{2}{s^{2}}\right)-\frac{1}{4 s}\right] d s \rightarrow \infty \text { as } t \rightarrow \infty,
\end{aligned}
$$

respectively, so equation (2.31) is oscillatory by Theorem 3 .

If we let $\beta=3, \gamma>4$ and $\rho(t)=t$. Then conditions (2.24) and (2.25) become

$$
\begin{aligned}
& \int_{4}^{t}\left[4 s^{\gamma-1}\left(1-\frac{4}{s^{2}}-\frac{4}{3 \sqrt{3} s}\right)^{3}-\frac{s}{8}\right] d s \rightarrow \infty \text { as } t \rightarrow \infty, \\
& \int_{4}^{t}\left[32 s^{\gamma-5}\left(\frac{3 \sqrt{3}-1}{3 \sqrt{3}}-\frac{2}{s^{2}}\right)^{3}-\frac{1}{4 s}\right] d s \rightarrow \infty \text { as } t \rightarrow \infty,
\end{aligned}
$$

respectively, so equation (2.31) is oscillatory by Theorem 2 .

Finally, we let $\beta=3, \gamma=9$, and $p_{2}(t)=t^{3 / 2}$. Taking $B=(\beta-1) \beta^{\frac{\beta}{1-\beta}}=$ $2 \cdot 3^{-3 / 2}$, it is not hard to see that

$$
g_{2}(t) \leq B t^{-9 / 4}, \quad g_{2}^{*}(t) \leq B t^{-9 / 4}, \quad Q^{*}(t) \geq t^{3 / 2}-(B / 2) t^{-5 / 4}>0
$$

and

$$
Q_{1}(t) \geq t^{3 / 2}-B t^{-1 / 4}>0 .
$$

With $\rho(t) \equiv 1$, it is easy to see that conditions (2.3) and (2.4) are satisfied. Thus, by Theorem 1, equation (2.31) is oscillatory.

Remark 1. The results of this paper are presented in a form that should make it easy to extended to higher order equations. It would also be of interest to use the approach here to study equation (1.1) with $\alpha>1$, i.e., equation (1.1) with a superlinear neutral term.

\section{References}

[1] R.P. Agarwal, M. Bohner, T. Li and C. Zhang. Oscillation of second order differential equations with a sublinear neutral term. Carpathian J. Math., 30:16,2014 . 
[2] L. Erbe, T. Hassan and A. Peterson. Oscillation criteria for nonlinear functional neutral dynamic equations on time scales. J. Diff. Eqn. Appl., 15(11-12):10971116, 2009. https://doi.org/10.1080/10236190902785199.

[3] L. Erbe, A. Peterson and P. Rehak. Comparison theorems for linear dynamic equations on time scales. J. Math. Anal. Appl., 275(1):418-438, 2002. https://doi.org/10.1016/S0022-247X(02)00390-6.

[4] S.R. Grace, R.P. Agarwal, M. Bohner and D. O'Regan. Oscillation of second order strongly superlinear and strongly sublinear dynamic equations. Comum. Nonlinear Sci. Numer. Stimul., 14(8):3463-3471, 2009. https://doi.org/10.1016/j.cnsns.2009.01.003.

[5] S.R. Grace, R.P. Agarwal, B. Kaymakalan and W. Sae-jie. Oscillation theorems for second order nonlinear dynamic equations. Appl. Math. Comput., 32(1):205218, 2010. https://doi.org/10.1007/s12190-009-0244-7.

[6] S.R. Grace, R.P. Agarwal and D. O'Regan. A selection of oscillation criteria for second order differential inclusions. Appl. Math. Letters, 22(2):153-158, 2009. https://doi.org/10.1016/j.aml.2008.01.006.

[7] S.R. Grace, M. Bohner and R.P. Agarwal. On the oscillation of second order half-linear dynamic equations. J. Difference Eqn. Appl., 15(5):451-460, 2009. https://doi.org/10.1080/10236190802125371.

[8] S.R. Grace, E. Akın and M. Dikmen. On the oscillation of second order nonlinear neutral dynamic equations with distributed deviating arguments on time scales. Dynam. Systems Appl., 23:735-748, 2014.

[9] G.H. Hardy, I.E. Littlewood and G. Polya. Inequalities. Cambridge University Press, 1959.

[10] C.G. Philos. On the existence of nonoscillatory solutions tending to zero at $\infty$ for differential equations with positive delays. Arch. Math. (Basel), 36(1):168-178, 1981. https://doi.org/10.1007/BF01223686. 